(C)2006 IEEE. Personal use of this material is permitted. However, permission to reprint/republish this material for advertising or promotional purposes or for creating new collective works for resale or redistribution to servers or lists, or to reuse any copyrighted component of this work in other works must be obtained from the IEEE.

This material is presented to ensure timely dissemination of scholarly and technical work. Copyright and all rights therein are retained by authors or by other copyright holders. All persons copying this information are expected to adhere to the terms and constraints invoked by each author's copyright. In most cases, these works may not be reposted without the explicit permission of the copyright holder.

The paper orginally appeared as:

Conventional and wavelet coherence applied to sensory-evoked electrical brain activity Klein, A.; Sauer, T.; Jedynak, A.; Skrandies, W.;

Biomedical Engineering, IEEE Transactions on

Volume 53, Issue 2, Feb. 2006 Page(s):266 - 272

Digital Object Identifier 10.1109/TBME.2005.862535 


\title{
Conventional and Wavelet Coherence Applied to Sensory-Evoked Electrical Brain Activity
}

\author{
Alexander Klein, Tomas Sauer, Andreas Jedynak, and Wolfgang Skrandies*
}

\begin{abstract}
The use of coherence is a well-established standard approach for the analysis of biomedical signals. Being entirely based on frequency analysis, i.e., on spectral properties of the signal, it is not possible to obtain any information about the temporal structure of coherence which is useful in the study of brain dynamics, for example. Extending the concept of coherence as a measure of linear dependence between realizations of a random process to the wavelet transform, this paper introduces a new approach to coherence analysis which allows to monitor time-dependent changes in the coherence between electroenecphalographic (EEG) channels. Specifically, we analyzed multichannel EEG data of 26 subjects obtained in an experiment on associative learning, and compare the results of Fourier coherence and wavelet coherence, showing that wavelet coherence detects features that were inaccessible by application of Fourier coherence.
\end{abstract}

Index Terms-Coherence, EEG topography, Fourier transform, learning, wavelet analysis.

\section{INTRODUCTION}

$\mathbf{C}$ OHERENCE is a well-established standard tool to analyze the linear relation between two signals by determining the correlation between their spectra. However, any analysis based entirely on spectral methods, hence on the Fourier transform, must ignore any temporal structure of the signal beyond phase information and so coherence cannot give any information on dynamically varying or "short time" dependence between the signals. In questions from physiology which concern brain dynamics, on the other hand, an additional temporal resolution is essential and necessitates the replacement of classical Fourier coherence by other methods. There have been successful attempts to adapt Fourier-based methods to short time signals, for example by means of orthonormal sliding windows as in [1]-[3], which are similar to the classical Gabor transform, developed in 1946 for the analysis of sound signals, e.g., [4]. In this paper, however, we incorporate a tool offering even more flexibility which has been developped during the last two decades, namely wavelets. To overcome many of the shortcomings of signal analysis based on the

Manuscript received November 17, 2004; revised May 30, 2005. This work was supported in part by the Deutsche Forschungsgemeinschaft, DFG under Grant SK 26/8-3. This study was performed at Justus-Liebig-University Gießen. Asterisk indicates corresponding author.

A. Klein and T. Sauer are with the Institute of Mathematics, D-35392 Gießen, Germany (e-mail: Alexander.Klein@math.uni-giessen.de; Tomas.Sauer@math.uni-giessen.de).

A. Jedynak is with the Institute of Physiology, D-35392 Gießen, Germany.

*W. Skrandies is with the Institute of Physiology, Aulweg 129, D-35392 Gießen, Germany (e-mail: Wolfgang.Skrandies@physiologie.med.unigiessen.de).

Digital Object Identifier 10.1109/TBME.2005.862535
Fourier transform, which are caused by essentially neglecting time resolution for the sake of perfect frequency resolution (i.e., the time-frequency atoms are the exponentials $e^{-i \xi \cdot}$ ), the wavelet transform has been established as an important technique in time-frequency analysis, e.g., [4]. Wavelets combine high temporal resolution with good frequency resolution and offer a reasonable balance between these parameters. Using, in addition, complex wavelets for signal analysis then yields the following two desirable effects simultaneously.

- Amplitude and phase information are extracted from the analyzed signal [4].

- Time is kept as an additional parameter, and the variation of coherence over time can be measured as well.

Although the phase information resulting from the wavelet transform is currently not fully understood, and is largely dependent on the specific wavelet, it has the useful property of being linear in time for sinusoidal signals and can, thus, be applied for computing coherence.

Wavelet based coherence and bicoherence for example, have already been used in other scientific contexts such as plasma physics [5] and aerodynamics [6]. However, the methods employed in these fields are not suitable for application to electroencephalographic measurements (EEG), because the signal to noise ratio in electric brain activity is too low to reliably analyze single events. This paper presents a new method for computing wavelet coherence, taking into account the traditional practice in neurophysical research - repetition and ensemble averaging [7]. We note that in the present study we are concerned with time-locked electrophysiological signals although there are other ways of data analysis. In addition to presenting the theoretical basis of wavelet coherence we will illustrate neurophysiological results obtained in an experiment where learning induces the coupling of different brain regions.

\section{Coherence - The Normalized Cross-SPeCtrum}

Many components of the EEG are of periodic nature, with alpha spindles being only one prominent example. Consequently, the most natural approach to detect similarities between two channels in the EEG is to look for them in the frequency domain, and in fact this has been done extensively using the normalized cross-spectrum, also known as coherence, which is defined as follows:

Let $x$ and $y$ be two ergodic and stationary signals [8]. Let $S_{x x}$ and $S_{y y}$ denote the Fourier transform of the respective autocorrelation function, which is also called autospectral density or autospectrum, and let $S_{x y}$ denote the Fourier transform of their cross-correlation function, which is also called cross-spectral 
density or cross spectrum. The magnitude squared coherence (furtherly referred to as coherence) $\gamma_{x y}^{2}(f)$ of the two signals at a given frequency $f \in \mathbb{R}$ is then defined as (see [8])

$$
\gamma_{x y}^{2}(f):=\frac{\left|S_{x y}(f)\right|^{2}}{S_{x x}(f) \cdot S_{y y}(f)}
$$

This definition, however, simple and elegant it is, causes some difficulties in practice, the most serious of which is the need to require stationarity. Under this assumption, the classical and usual way to estimate the above spectra is to break both signals into $n$ parts of equal size, $x_{k}$ and $y_{k}$, and estimate the autospectra and the cross-spectrum as a time average by means of the Fourier transform as

$$
\tilde{S}_{x y}(f):=\frac{1}{n-1} \sum_{k=1}^{n} \overline{\hat{x}_{k}(f)} \cdot \hat{y}_{k}(f) \approx S_{x y}
$$

leading to the well-known estimation for coherence [8]

$$
\tilde{\gamma}_{x y}^{2}(f)=\frac{\left|\sum_{k=1}^{n} \overline{\hat{x}_{k}(f)} \cdot y_{k}(f)\right|^{2}}{\sum_{k=1}^{n}\left|\hat{x}_{k}(f)\right|^{2} \cdot \sum_{k=1}^{n}\left|\hat{y}_{k}(f)\right|^{2}},
$$

where, as usual, the symbol $\bar{x}$ denotes the complex conjugate of $x$. As mentioned above, the (unavoidable) condition that the computed quantity $\tilde{\gamma}_{x y}^{2}(f)$ approximates the coherence $\gamma_{x y}^{2}(f)$ requires the signals to be stationary, which is usually not at all ensured in experiments analysing electrical brain activity. To overcome this problem, we make use of repetition:

Many neurophysical experiments present the same stimulus several times to a subject in order to enhance the signal-to-noise ratio, for example by signal averaging, which is based on the implicit assumption that the noise is a zero-mean random variable independent of repetition. This of course does not neglect the potential importance of spontaneous neurophysiological events. Hence, such an experiment can be thought of as producing an ensemble of measurements of the same random process, and an ensemble average can be computed as soon as the repetitions of the experiment can be located in time; for example, in the experiment from [7] the exposition of the visual stimulus is marked in the data. The fundamental advantage of ensemble averaging over time averaging is that stationarity of the signal is no longer needed as a prerequisite to get an estimator for coherence. Instead, it is sufficient to rely solely on ergodicity, which means that the individual measurements in the ensemble are each assumed to be statistically representative for the random process analyzed [8]. Moreover, this point of view leads directly to the analysis of relevant data only: many of the changes that experiments induce in the EEG are very small, giving little hope of finding them with a method based on time averaging. On the other hand, when restricting the attention only to the relevant time frames (in our main example from [7], the ensemble consisting of every $250 \mathrm{~ms}$ of data after stimulus onset), two different electrodes yield two data sets $X=\left(x_{1}, \ldots, x_{n}\right)$ and $Y=\left(y_{1}, \ldots, y_{n}\right)$, both ordered in time, and each containing an ensemble of $n$ recordings of a certain neurophysical experiment in a single subject. These recordings can now be safely interpreted as repetitions of realizations of the same random process and the autospectra and the cross-spectrum can then be estimated as an average over the ensemble of recordings as in (2), leading again to (3) as an estimate for coherence. In other words: while the formulae remain the same, their interpretation changes significantly as they now deal with two entirely different concepts of data acquisition.

When rewriting $\tilde{\gamma}_{x y}^{2}$ in terms of polar coordinates

$$
\tilde{\gamma}_{x y}^{2}=\frac{\left|\sum_{k=1}^{n}\right| \hat{x}_{k} \hat{y}_{k}\left|e^{\imath\left(\arg \left(\hat{y}_{k}\right)-\arg \left(\hat{x}_{k}\right)\right)}\right|^{2}}{\sum_{k=1}^{n}\left|\hat{x}_{k}\right|^{2} \cdot \sum_{k=1}^{n}\left|\hat{y}_{k}\right|^{2}}
$$

it becomes clear how the coherence estimator works - both, amplitude and phase information are evaluated with regard to their contribution to linear dependence.

Coherence is very sensitive to fluctuations of linearity in phase, relatively less so to nonlinear fluctuations of amplitude and completely insensitive to linear fluctuations in amplitude. How much both types of linearity contribute to the final measure, however, remains somewhat unclear and, therefore, some authors have argued that amplitude should not contribute to the final measure at all, using a "phase locking value" [9] instead, which is essentially the square root of (4) with all the moduli set to 1 .

\section{WAVELET COHERENCE}

\section{A. The Wavelet Transform and its Properties}

The wavelet transform is one of the standard means to perform signal analysis in time and frequency. Basically, it is achieved by correlating the signal with a wavelet $\psi \in L_{2}(\mathbb{R})$ that satisfies the admissibility condition

$$
\int_{\mathbb{R}} \frac{|\hat{\psi}(f)|^{2}}{|f|} d f<\infty .
$$

Recall, e.g., from [4, Theorem 4.3] that the admissibility condition (5) ensures the invertibility of the wavelet transform and is only slightly sharper than the "wave" condition $\widehat{\psi}(0)=0$.

Since these are only very general prerequisites, it seems reasonable to require additional properties that are useful in the context of coherence analysis:

- Since the coherence estimator is very sensitive to phase information, the wavelet should be complex.

- The spectrum of the wavelet should be unimodal, so as not to move too far away from the traditional context of frequency bands.

- $\psi \in L_{2}(\mathbb{R})$ necessitates that $\lim _{|t| \rightarrow \infty} \psi(t)=0$, and $\lim _{|f| \rightarrow \infty} \hat{\psi}(f)=0$. It is, however, not clear if there is a particular rate of decay that is more desirable than others in either domain, at least not in the context of coherence.

Many wavelets have already been developed for different applications, and new ones can easily be constructed to fit special needs. While most of those methods are concerned with the construction of real valued wavelets, there has been some recent work devoted to the construction of complex valued ones [10]. 
The wavelet transform itself is defined as (see [11])

$$
W_{\psi} f(a, b):=\int_{\mathbb{R}} \overline{f(t)} \psi\left(\frac{t-b}{a}\right) d t
$$

with $a$ representing reciprocal frequency, and $b$ representing time. This way, the time resolution clearly improves for higher frequencies.

In practice, this expression is evaluated very easily and efficiently by means of the fast Fourier transform as some basic properties of the Fourier transform yield the identity

$$
W_{\psi} f(a, b)=\left(\bar{f} \star \psi\left(\frac{\dot{a}}{a}\right)\right)(-b)=\left(\overline{\hat{f}} \cdot \hat{\psi}\left(\frac{\dot{a}}{a}\right)\right)^{\vee}(-b)
$$

which has significant computational advantages compared to evaluating (6) directly, for example by means of quadrature formulae. For further details see [4] and [11] and the references therein.

\section{B. Wavelet Coherence}

As mentioned above, coherence is most strongly influenced by linearity in phase, which makes it necessary to use complex wavelets in order to compute a meaningful wavelet coherence. A conceptually simple wavelet that has already proved useful for coherence and bicoherence analysis in [5], and in its real form in [6], is the Morlet wavelet, which is defined as

$$
\psi_{M}(t):=e^{-t^{2}} \cdot e^{-\imath 2 \pi t}
$$

This modulated Gaussian (see Fig. 1) has a simple and very smooth spectrum that allows for easy interpretation of the results achieved.

The above references use wavelet coherence or bicoherence to detect coherent events which appear at completely random times. They achieve their goal by defining the wavelet equivalent of cross- and autospectra by means of integration over a short period of time. There is no standard approach to computing wavelet coherence, however. The authors in [12] use still different formulae, and none of the above approaches takes into account any repetitive nature of the experiment, in contrast to our situation, where we expect that the same neurophysiological process is triggered by the stimulation over and over again.

To detect phase correlation due to the presented stimuli, we define the estimated wavelet coherence as a measure of linear dependence between two sets, i.e., an ensemble of wavelet transforms, where $(a, b)$ remain fixed

$$
\tilde{\Gamma}_{u v}^{2}(a, b)=\frac{\left|\sum_{k=1}^{n} \overline{W_{\psi} u_{k}(a, b)} \cdot W_{\psi} v_{k}(a, b)\right|^{2}}{\sum_{k=1}^{n}\left|W_{\psi} u_{k}(a, b)\right|^{2} \cdot \sum_{k=1}^{n}\left|W_{\psi} v_{k}(a, b)\right|^{2}} .
$$

\section{An Application of Wavelet Coherence to Artificial Data}

In order to demonstrate in which way wavelet and Fourier coherence can differ in the quality of their results, we consider the following example: A time series containing spindle-like

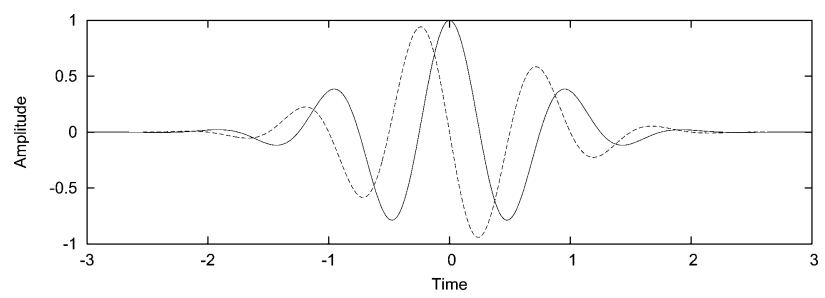

Fig. 1. The Morlet wavelet is a complex wavelet. The solid curve represents its real part, while the dashed one represents its imaginary part.

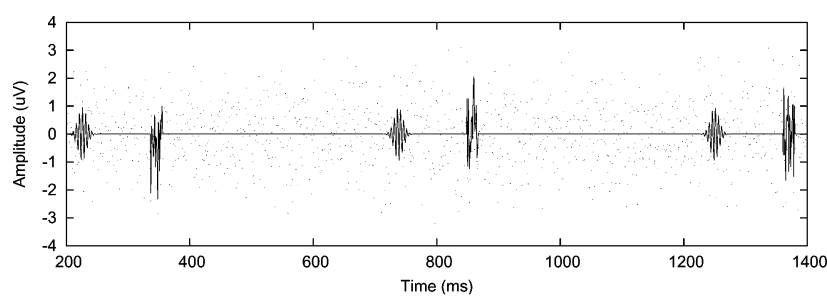

(a) General Structure

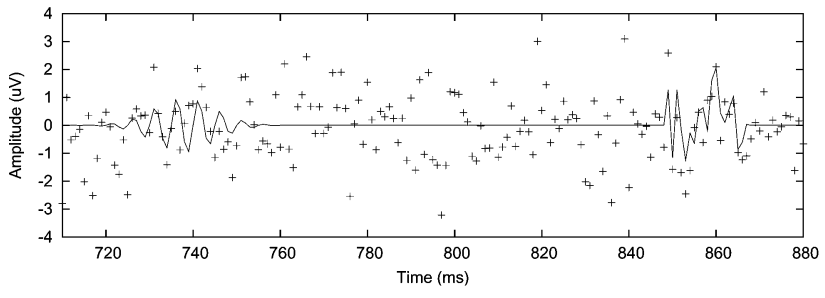

(b) Detail

Fig. 2. Spindle-like structures and noise bursts (solid line) embedded in a white noise floor (dots). Note that the peak amplitude of the spindles is relatively small compared to that of the noise, and they are uniquely localized in time. Note different times scales: (a) $1200 \mathrm{~ms}$; (b) $170 \mathrm{~ms}$.

structures with a center frequency of $100 \mathrm{~Hz}$ and short bursts of $N(0,1)$-noise with a duration of $20 \mathrm{~ms}$ is generated (Fig. 2). The first spindle appears centerd at $226 \mathrm{~ms}$, the first burst appears centerd at $346 \mathrm{~ms}$, and the time difference between adjacent spindles as well as adjacent noise bursts is $512 \mathrm{~ms}$. This time series is embedded in two channels of independent $N(0,1)$-noise. To compare Fourier- and wavelet coherence, both were estimated with the same size and number of blocks, namely 128 blocks of 512 samples with a sampling rate of one sample per ms. While Fourier coherence hardly detects anything at all (even if it is computed in the framework of ensemble averages as well), with the spindles showing up as a very small peak around $100 \mathrm{~Hz}$ [Fig. 3(a)], wavelet coherence identifies the spindles as a large peak at a center-latency of about $226 \mathrm{~ms}$ at $100 \mathrm{~Hz}$, and the noise bursts as a broad band of coherence covering almost the entire frequency range at a center-latency of $346 \mathrm{~ms}$. [Fig. 3(b)]. The drop-off in wavelet coherence for the noise bursts is due to the reduced temporal resolution at low frequencies. Though not shown in the example, wavelet coherence also performed similarly well in the detection of other waveforms, e.g., rectangular and triangular ones. Of course, Fourier coherence is based on the classic periodogram estimate and, thus, cannot be expected to perform well in this setting, but also the generic Morlet wavelet bears little resemblance to the noise bursts and still detects them well. 


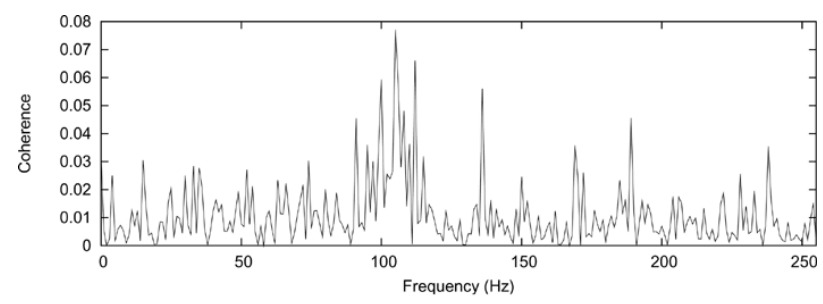

(a)

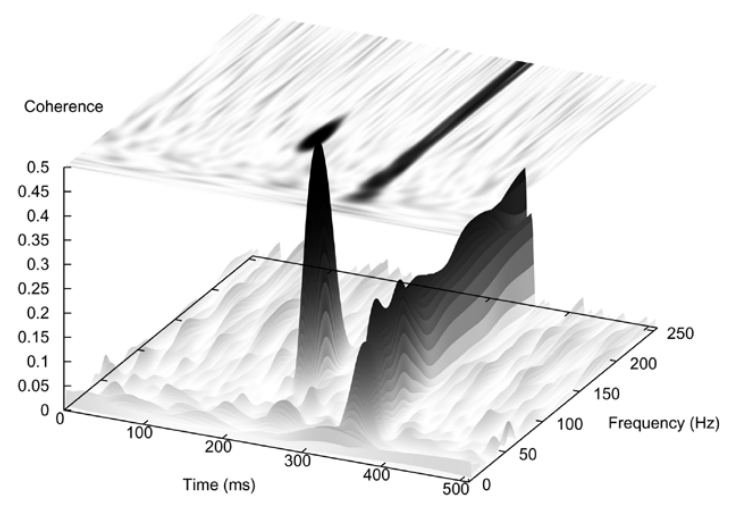

(b)

Fig. 3. (a) Fourier based coherence - even with well-tuned parameters - does hardly react to the spindles and virtually not at all to the noise bursts shown in Fig. 2. Although the spindles appear as a small peak around $100 \mathrm{~Hz}$, the measured coherence is still very small. (b) Wavelet coherence, on the other hand, clearly detects spindles and noise-bursts at the correct latencies and frequencies.

\section{The Statistics OF COHERENCE}

For Fourier coherence, the statistics of the estimator are known under the assumption that at least one channel carries Gaussian white noise, see for example [13]-[15]. However, the EEG is far from being a Gaussian white process, and consequently, we can only employ nonparametric tests. As of yet, we do not know of any results on the analytical properties of the statistics of wavelet coherence, which are very likely to depend fundamentally on the underlying wavelet. The statistical results displayed were achieved with two-sided Mann-Whitney (intergroup) and Wilcoxon (intragroup) tests (see [16]). The significance level of the tests was $p=0.05$ for each tail. All results were achieved with the R-package for statistical computing [17]. We note that we are concerned with exploratory data analysis seeking some structure in the results' space. Thus, exact significance levels should be taken as some guidance.

\section{An Application of WAVElet COHERENCE to EXPERIMENTAL DATA}

Wavelet coherence was applied to a set of experimental data stemming from a total of 26 adult human subjects (16 females, 10 males, 19-31 years of age). The study was concerned with associative learning, and dealt with the question of how different brain regions are functionally coupled after learning the association between stimuli of different sensory modalities. As visual stimuli we employed Landolt-Rings (DIN 58 220; standard optotypes for the determination of visual acuity) and full rings. The presentation of Landolt-rings ("conditioned stimulus": CS+) was paired with an electric stimulus ("unconditioned stimulus":

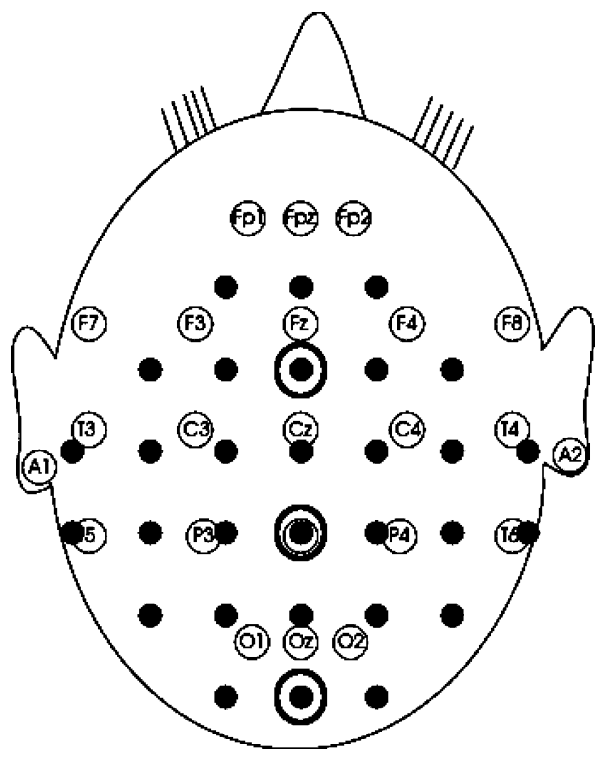

Fig. 4. Electrode positions: The black dots indicate the electrode positions used in our experiment. The labels refer to the electrode positions of the International 10-20 System. The results of the coherence analysis for the posterior pair of electrodes marked by circles are shown in Fig. 5.

UCS) applied to the left median nerve following the visual stimulus after $500 \mathrm{~ms}$. Neutral visual control stimuli were full circles ("conditioned stimulus without reinforcement": CS-) that were not paired with the UCS. Identical stimuli were used in a control experiment where, however, there was no contingency between stimuli. For both groups, the experiment was divided into an acquisition- and an extinction phase. The subjects were assigned to a conditioning or control group at random, and groups did not differ with respect to age and gender. The EEG was recorded from 30 electrodes covering the whole scalp (see Fig. 4); the bandpass was from 0.16 to $70 \mathrm{~Hz}$, and a sampling rate of 500 $\mathrm{Hz}$ was employed; for further methodological details we refer to [18].

The basic idea of this experiment was that after successful learning an enhancement of coherence between brain regions processing visual and somatosensory stimuli could be expected. Thus, we can compare data obtained before and after learning as well as the effects induced in the group of "learners" and controls ("nonlearners"). From the experimental design we expect that changes in coherence are different in different pairs of recording channels. As a consequence of successful conditioning changes in coherence of electrodes overlying visual and somatosensory areas should be more prominent than with other pairs of electrodes.

The EEG recordings were analyzed for changes in coherence using Fourier and wavelet methods. For the analysis, only artefact-free episodes starting $12 \mathrm{~ms}$ before visual stimulus onset (512 ms before the possible electric stimulus), and ending 512 ms after the possible electric stimulus were used. In this setting, the Fourier based method allowed a per group and visual stimulus comparison of coherence before and after the possible electric stimulus, revealing a highly structured pattern of significant changes, especially in interhemispheric coherence over the parieto-occipital regions, depending on group and type of visual stimulus (see [19]), but only for the $\delta-, \theta-$, and $\alpha$-bands. 


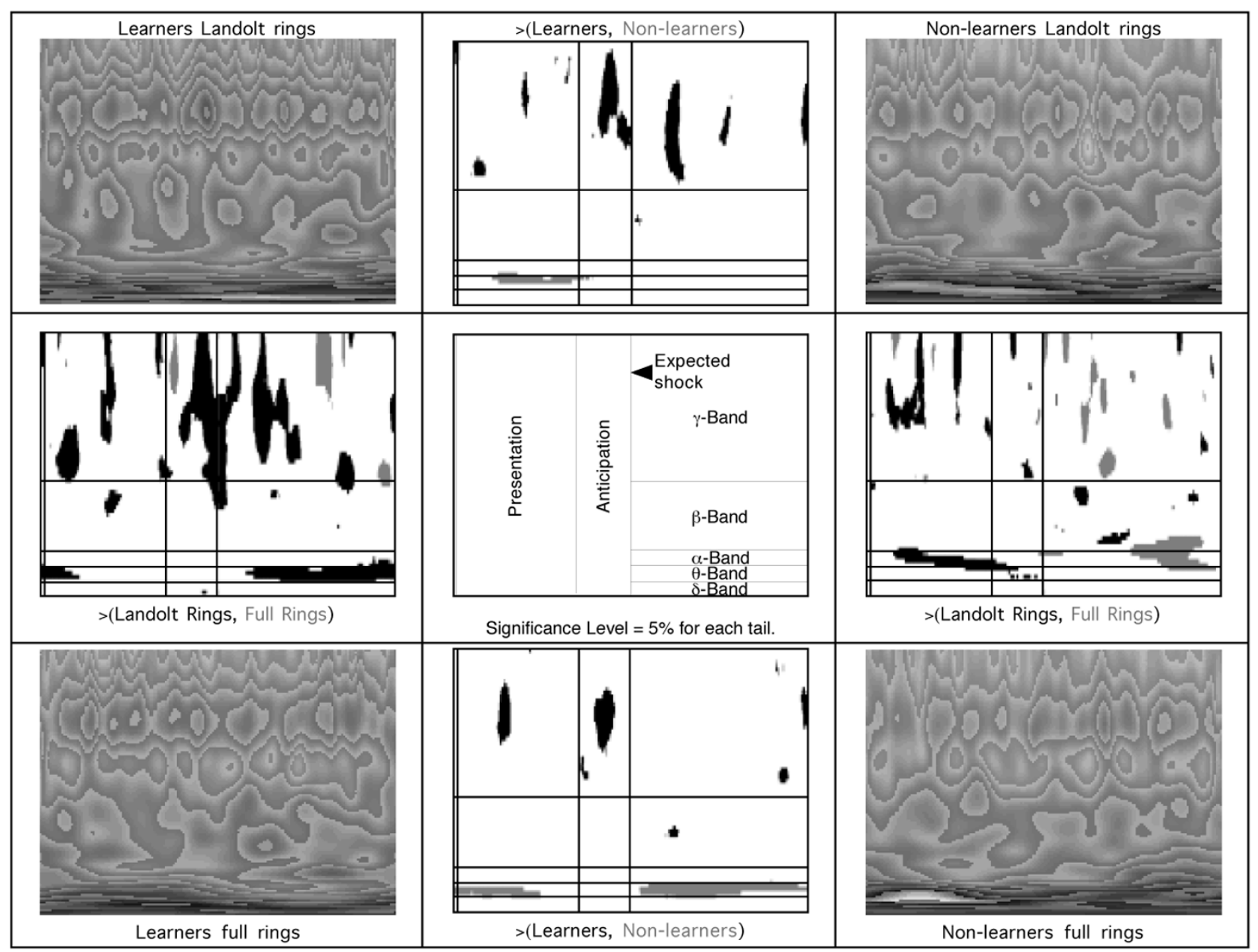

Fig. 5. Results of the analysis of wavelet coherence for the posterior pair (see Fig. 4) of electrodes. The diagrams in the corners show the averaged wavelet coherence maps for the group of "learners" (left) and "nonlearners" (right), elicited by Landolt rings (upper part) or full rings (lower part). The other diagrams illustrate the results of non parametric Wilcoxon tests (pairwise where adequate) of corresponding coherence distributions. This results in areas of significant difference of coherence induced by learning or group. Time from -512 to $+512 \mathrm{~ms}$ relative to the moment of (potential) electric stimulus (UCS), frequency between 0 and $72 \mathrm{~Hz}$. Conventional EEG frequency bands as indicated (see inset in the center). Frequency bands are: $\delta$-band: $0-4 \mathrm{~Hz}, \theta$-band: $4-8 \mathrm{~Hz}, \alpha$-band: $8-12 \mathrm{~Hz}, \beta$-band: $12-30 \mathrm{~Hz}$, and $\gamma$-band: $\geq 30 \mathrm{~Hz}$.

For analysis we selected two of the possible 435 pairs of electrodes. They were formed by the three electrodes which are marked in Fig. 4. The posterior pair consisted of the electrode located at $\mathrm{Pz}$, while the other was $10 \%$ posterior to $\mathrm{Oz}$ of the International 10-20 System. Thus, these electrodes were in the vicinity of visual and somatosensory areas of the human brain, and they were also close to the pairs of electrodes that are known to be connected in this type of process and also showed coherence changes in [20]. The anterior pair consisted of the electrode at $\mathrm{Pz}$ again, while the other electrode was located between $\mathrm{Cz}$ and Fz. Since we expect less changes in coherence at anterior brain areas, this pair of electrodes served as additional control.

Instead of comparing the preshock and postshock coherence, wavelet coherence allowed the statistical comparison of coherence between groups of subjects for a fixed type of visual stimulus, and between the experimental and control group for both types of visual stimuli.

Fig. 5 illustrates the mean results based on the average of the individual coherence values of all subjects. The diagrams in the four corners show the average wavelet coherence in the extinction-phase (in which no actual electric stimulation occurred) for a given group and stimulus condition, plotted as a function of poststimulus time and signal (EEG) frequency. The other plots illustrate the results of the statistical comparison of different conditions (areas in time and frequency where coherence was significantly different). The central inset describes the parameters: The time axis ranges from $512 \mathrm{~ms}$ before to $512 \mathrm{~ms}$ after the moment at time "0" when the electric stimulus (UCS) was applied in the learning phase and, thus, could be expected to be anticipated in the extinction phase. The interval before the UCS can be divided into a "presentation" phase where the visual stimulus occurred, and an "anticipation" phase where the UCS was expected. As one might expect, after successful learning the anticipation occurs only in the group of "learners" and only with the Landolt stimuli. The labeling of different frequency ranges was made according to the classical frequency bands used in EEG research [7].

The top row represents the data of the "learners" (left) and "nonlearners" (right) for whom brain activity was elicited by Landolt rings. The bottom row shows corresponding data evoked by the control stimuli (full rings). Differences between the data evoked by Landolt or full rings are interpreted as 
correlate of learning, since an association between visual and somatosensory stimuli is formed only for the Landolt rings but not for the control stimuli. The corresponding statistical test rests on the comparison of wavelet coherences in the "learners" group (upper left vs. lower left). Learning effects are seen during the anticipation phase as a significant coherence increase in the $\gamma$-band and after the electrical stimulus there is an increase in coherence in the $\theta$-band, see the statistical results on the left of Fig. 5. In the groups of "nonlearners" no such differences occur which supports our interpretation that the changes in coherence observed in the experimental group were induced by associative learning. The difference between the subject groups was also confirmed by testing the number of significant differences before and after conditioning in the experimental and in the control subjects. This turned out to be highly significant $\left(\chi^{2}=688, \mathrm{p}<0.00001\right)$ In a similar line, there are several statistically significant differences between "learners" and "nonlearners" where increased coherences are observed in the $\gamma$-band and decreased values occur in the $\theta$-band. We also note that the analysis of the anterior pair of electrodes yielded a much smaller number of significant effects in the group of "learners" (2551 vs. 5394 significant data points) supporting our assumption that this region is less affected by associative learning.

Unlike Fourier coherence which gave no indication for the presence of interesting features in the $\gamma$-band, wavelet coherence reveals temporally structured differences of coherence in the $\gamma$-band in almost all compared pairs of stimuli, the only notable exception being the comparison of coherence for full and Landolt rings in the control group, where, by the design of the experiment, no significant differences should appear.

\section{DISCUSSION}

The example and the application to real experimental data both suggest that wavelet coherence offers new means for the analysis of interdependence between brain signals as far as the detection of short-lived coherent events is concerned. This becomes especially obvious in the $\gamma$-band, where wavelet coherence detects many features that classical Fourier coherence did not find at all. The fact that these features did not occur in the control group strongly indicates that they are real features and no artefacts.

Some of the problems with wavelet coherence that remain to be solved are the visualization of the huge amount of data and the unknown statistical properties of wavelet coherence, allowing only for very weak and general statistical tests. One minor drawback of wavelet coherence is its inability to detect coherence when the delay between the two signals becomes large relative to their duration and frequency. This is due to the fast temporal decay of the wavelet, but can be circumvented, for example, by using separate time parameters $b_{1}, b_{2}$ for each of the two wavelet transforms in the definition of $\tilde{\Gamma}^{2}$. Unfortunately, this also leads to yet another dimension for the results, making their visualization and interpretation even more difficult.

In addition, the use of wavelet based coherence allows us to detect and describe the dynamical change of coherence over time which turned out to be in accordance with the layout of the experiment. Of course, any method with time and frequency components based on the concept of ensemble averages can be expected to give more information than standard coherence as the latter ignores the time aspect completely. We expect the methods used in [1] and [2] would probably give better results than classic Fourier-based coherence, but a fair comparison of these methods to ours would require to apply identical implementations on identical data which would be most interesting, but is beyond the scope of this paper. Still another approach would be to consider a windowed Fourier transform which is, however, not without problems: besides the drawback of unavoidable artefacts stemming from the well-known effect of window-leakage, the short time segments will provide only a poor frequency resolution. Since the Morlet wavelet was not specially designed for EEG-applications, it remains to be explored in which way the choice of an optimally designed wavelet can further improve the performance of wavelet coherence.

\section{REFERENCES}

[1] Y. Xu, S. Haykin, and R. J. Racine, "Multiple window time-frequency distribution and coherence of eeg using slepian sequences and hermite functions," IEEE Trans. Biomed. Eng., vol. 46, no. 7, pp. 861-866, Jul. 1999.

[2] E. G. Lovett and K. M. Ropella, "Time-frequency coherence analysis of atrial fibrillation termination during procainamide administration," Ann. Biomed. Eng., vol. 25, pp. 975-984, 1997.

[3] M. Bayram and R. Baraniuk, "Multiple window time frequency analysis," in Proc. IEEE-SP Int. Symp. Time-Frequency and Time-Scale Analysis, Jun. 1996, pp. 173-176.

[4] S. Mallat, A Wavelet Tour of Signal Processing. New York: Academic, 1999.

[5] B. P. van Milligen, E. Sanchéz, C. Hidalgo, B. Brañas, B. Carreras, and L. García, "Wavelet bicoherence: A new turbulence analysis tool," Phys. Plasmas, vol. 2, no. 8, pp. 3017-3032, 1995.

[6] K. Gurley, T. Kijewski, and A. Kareem.. (2003) Higher Order Correlation Detection in Nonlinear Aerodynamic Systems Using Wavelet Transforms. [Online]. Available: http://www.nd.edu/ nathaz/publications/wind/icossar2.pdf

[7] W. Skrandies, "Electroencephalogram (EEG) topography," in The Encyclopedia of Imaging Science and Technology, J. Hornak, Ed. New York: Wiley, 2002, vol. 1, pp. 198-210.

[8] J. S. Bendat and A. G. Piersol, Engineering Applications of Correlation and Spectral Analysis, 2nd ed. New York: Wiley, 1993.

[9] J.-P. Lachaux, E. Rodriguez, J. Martinerie, and F. J. Varela, "Measuring phase synchrony in brain signals," Hum. Brain Mapp., vol. 8, pp. 194-208, 1999.

[10] X.-P. Zhang, M. Desai, and Y.-N. Peng, "Orthogonal complex filter banks and wavelets: Some properties and design," IEEE Trans. Signal Process., vol. 47, no. 4, pp. 1039-1048, Apr. 1999.

[11] I. Daubechies, Ten Lectures on Wavelets. Philadelphia, PA: SIAM, 1992, vol. 61, CBMS-NSF Regional Conference Series in Applied Mathematics.

[12] S. Sello and J. Bellazzini.. Wavelet Cross-Correlation of Turbulent Mixing from Large-Eddy-Simulations. [Online]. Available: http://arxiv.org/ps/physics/0 003029

[13] D. R. Brillinger, "Time series," in Data Analysis and Theory. Philadelphia, PA: SIAM, 2001.

[14] G. C. Carter, C. Knapp, and A. H. Nuttall, "Estimation of the magnitude-squared coherence function via overlapped fast Fourier transform processing," IEEE Trans. Audio Electroacoust., vol. EA-21, pp. 337-344, 1973.

[15] A. Nuttall, "Invariance of distribution of coherence estimate to second channel statistics," IEEE Trans. Acoust., Speech, Signal Process., vol. ASSP-29, no. 2, pp. 120-122, Feb. 1981.

[16] S. Siegel, Nonparametric Statistics for the Behavioral Sciences. New York: McGraw-Hill, 1988.

[17] “R: A Language and Environment for Statistical Computing," R Development Core Team, R Foundation for Statistical Computing, Vienna, Austria, ISBN 3-900 051-00-3, 2004. 
[18] W. Skrandies and A. Jedynak, "Associative learning in humans - Conditioning of sensory-evoked brain activity," Behav. Brain Res., vol. 107, pp. 1-8, 2000.

[19] A. Klein, "Mathematische Analyse assoziativer Lernvorgänge anhand von Phasenkohärenz im EEG” (in German), in Diplomarbeit. Gießen, Germany: Justus-Liebig-Universität Gießen, 2003.

[20] W. H. R. Miltner, C. Braun, M. Arnold, H. Witte, and E. Taub, "Coherence of gamma-band EEG activity as a basis for associative learning," Nature, vol. 397, pp. 434-436, 1999.

Alexander Klein received the diploma in mathematics from Justus-Liebig-University, Gießen, Germany, in 2003. Most of the results in this paper originate from his diploma thesis, supervised by T. Sauer and W. Skrandies.

Tomas Sauer studied mathematics and computer science at the University of Erlangen, Erlangen, Germany. He received Dr. rer. nat degree in 1993, the habilitation in 1998, both from the University in Erlangen.

Since 2000, he is Professor of Applied Mathematics and Scientific Computing, Justus-Liebig-University, Gießen, Germany.

He received the Heisenberg research fellowship from Deutsche Forschungsgemeinschaft.
Andreas Jedynak studied physics at the University of Freiburg, Freiburg, Germany. His 1994 doctoral thesis was on chaos analysis of human EEG. His research work is in multichannel EEG at University, Gießen.

Since 2001, he is Information System Administrator for the Eye Clinic at the University of Freiburg.

Wolfgang Skrandies studied physiological psychology and biology at University of Konstanz, Konstanz, Germany. In 1979, he received the Dr. rer. nat degree at the University of Konstanz in 1979 and the habilitation from the University of Gießen, Gießen, Germany, in 1986

His further research activities were at the University of Zurich, University of Rochester, the Max-Planck-Institute for Physiological and Clinical Research, Bad Nauheim and Frankfurt, Germany, and the University of Bielefeld, Bielefeld, Germany. Since 1991, he is Professor of Physiology at the Justus-Liebig-University, Gießen. 